\title{
Forming Limit Predictions for Single-Point Incremental Sheet Metal Forming
}

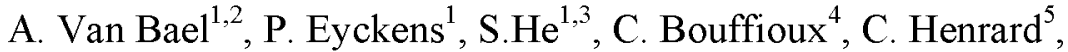 \\ A.M. Habraken ${ }^{5}$ J. Duflou ${ }^{6}$ and P.Van Houtte ${ }^{1}$ \\ ${ }^{1}$ Dept. MTM, Katholieke Universiteit Leuven, Kasteelpark Arenberg 44, B-3001 Heverlee, Belgium \\ ${ }^{2}$ Dept. IWT, Katholieke Hogeschool Limburg, Campus Diepenbeek, Agoralaan Gebouw B, bus 3 , \\ B-3590 Diepenbeek, Belgium \\ ${ }^{3}$ now at Technology Center, Baoshan Iron and Steel Co., Ltd., Fujin 655, 201900 Shanghai, China \\ ${ }^{4}$ Dept. MEMC, Vrije Universiteit Brussel, Pleinlaan 2, B-1050 Brussels, Belgium \\ ${ }^{5}$ Dept. ArGEnCo, Université de Liège, Chemin des Chevreuils 1, B-4000 Liège, Belgium \\ ${ }^{6}$ Dept. PMA, Katholieke Universiteit Leuven, Celestijnenlaan 300B, B-3001 Heverlee, Belgium
}

\begin{abstract}
A characteristic of incremental sheet metal forming is that much higher deformations can be achieved than conventional forming limits. In this paper it is investigated to which extent the highly non-monotonic strain paths during such a process may be responsible for this high formability. A Marciniak-Kuczynski (MK) model is used to predict the onset of necking of a sheet subjected to the strain paths obtained by finite-element simulations. The predicted forming limits are considerably higher than for monotonic loading, but still lower than the experimental ones. This discrepancy is attributed to the strain gradient over the sheet thickness, which is not taken into account in the currently used MK model.
\end{abstract}

Keywords: formability, Marciniak-Kuczynski model, finite-element simulations.

PACS: 81.05.Bx, 81.40.Ef, 81.40.Lm, 81.40.Np, 83.10.Ff, 83.10.Gr, 83.80.Ab.

\section{INTRODUCTION}

Single-point incremental forming (SPIF) has emerged in the past few years as a potential alternative to conventional sheet metal deep-drawing to meet the increasing need for rapid prototyping and small batch productions at low cost (see, e.g., review paper [1]). A smooth ended tool on a $\mathrm{CNC}$ machine is used to create a local indentation in a clamped sheet, and by moving the point of contact around the sheet according to a programmed tool path, 3-dimensional shapes can be formed. The process is characterized by highly localized deformations. Also, the achievable deformations are much larger than conventional forming limits [2]. Previous studies have revealed that during incremental forming the sheet material is subjected to highly non-monotonic, serrated strain paths [3]. In the present paper, it is investigated to which extent these strain paths may contribute to the increased formability. Forming limits are predicted with a modified version of a model that has been used for forming limit curves of aluminium alloys [4]. Various descriptions of the yield surface and the hardening behaviour are used, including a texture-based yield locus and a microstructure-based work hardening/softening model. 


\section{EXPERIMENTAL DATA}

A cold rolled and annealed aluminium AA3003-O sheet of $1.2 \mathrm{~mm}$ thickness is considered in the present study. A three-axis CNC vertical milling machine is used as the platform for the SPIF process. A sheet of $225 \times 225 \times 1.2 \mathrm{~mm}$ is clamped on a foursided steel fixture using a backing plate with circular orifice, in which cones with different wall angles are formed. During the process, a steel tool with a diameter of $10.0 \mathrm{~mm}$ follows a series of circular contours. After each contour, the tool moves $0.5 \mathrm{~mm}$ deeper in a stepwise fashion. This process is repeated until a partial cone with a depth of $40 \mathrm{~mm}$ is formed [5].

The limiting wall angle is found to be $72^{\circ}$. Cones up to $71^{\circ}$ were made without failure. One out of two experiments with $72^{\circ}$ cones resulted in a failed piece, as did the cone with a $73^{\circ}$ wall angle.

In order to characterize the material behaviour, tensile tests have been performed in a standard tensile test machine, while the bi-axial machine developed at the University of Liège has been used for plane strain and simple shear tests [6]. The experimental procedures to obtain the material parameters for various hardening models are documented in [3]. Also, the crystallographic texture of the initial sheet has been measured using X-ray diffraction on a plane specimen at $50 \%$ depth of the sheet. It shows a strong cube component $\{001\}<100>$, which is typical for recrystallisation textures, and weaker other components: S $\{123\}<412>$, Goss $\{011\}<100>$ and Brass $\{011\}<211>$. The homogenisation procedure described in [7] was used to derive the shape of the texture-based yield locus, starting from the crystallographic orientation distribution function and the Taylor theory while assuming $\{111\}<110>$ slip systems.

\section{NUMERICAL MODELLING}

\section{Finite-Element Simulations}

The three-dimensional elastic-plastic finite-element model described in [8] is used to simulate the single-point incremental forming of truncated cones with wall angles of $50^{\circ}$ and $73^{\circ}$. The simulations are performed with the commercial FEM package Abaqus/Standard. A $40^{\circ}$ pie of the blank is considered since a full model is computationally too demanding. The sheet is modelled with three layers of brick elements. The elastic-plastic material behaviour is assumed to be isotropic, using a von Mises yield criterion with the Swift-type hardening law $\sigma=184(\varepsilon+0.00196)^{0.224}$ (MPa). The strainpath history throughout the forming process was extracted for an element in the outer layer of the cone wall.

\section{Forming Limit Predictions}

A previously developed model for the prediction of forming limits [4] has been modified and extended in order to deal with the serrated strain paths that occur during incremental forming. It is based on the Marciniak-Kuczynski theory [9]. The method simulates the evolution of a pre-existing groove in a metal sheet which is being deformed according to a given strain path. For each imposed deformation increment, 
strain increments in the groove are calculated on the basis of geometrical compatibility and force equilibrium between the groove and the surrounding sheet material. In practice, this requires the solution of a set of two non-linear equilibrium equations. This is solved as a minimisation problem: the residual $R=f_{1}^{2}+f_{2}^{2}$ is minimised (with $f_{1}=0$ and $f_{2}=0$ the force equilibrium equations). In the previous software, the onset of necking was assumed to take place when the strain rate in the groove exceeds a critical limit (in particular when the thinning strain rate in the groove is more than 10 times that in the surrounding sheet). It was necessary to implement a second forming limit criterion to account for the possibility that the force equilibrium equations cannot be satisfied any more at the end of the (finite) increment. Further details will be presented at a forthcoming conference [10].

Three material models were considered for the forming limit calculations: isotropic plasticity with a von Mises yield locus and Swift-type hardening (i.e. the assumptions used for the finite-element simulations), an anisotropic texture-based yield locus in combination with isotropic hardening, and the texture-based yield locus combined with a microstructure-based anisotropic hardening/softening model. These models will be referred to as VON-ISO, TEX-IsO, and TEX-MIC, respectively, in this paper. Forming limits have been predicted for both serrated and monotonic strain paths.

\section{RESULTS AND DISCUSSION}

The strain-path histories obtained with the finite-element simulations of the truncated cones with wall angles of $50^{\circ}$ and $73^{\circ}$ are given in Fig. 1 for an element at the outer side of the cone wall. These show the evolutions of the major and minor principal true plastic strains in the plane of the sheet. Highly non-monotonic, serrated strain-path changes occur. The direction of the major principal strain slightly oscillates around the radial direction along the cone wall. Correspondingly, the minor principal strain in the plane of the sheet is approximately aligned with the circumferential direction of the cone, i.e. the direction of the tool movement. During the process the sheet is severely stretched along the radial direction, whereas near plane strain conditions apply along the circumferential direction. At the same time, the sheet thickness is severely reduced. At the end of the process, the simulated principal true strains along radial and circumferential directions are 0.434 and 0.016 , respectively, for the $50^{\circ}$ cone, and 1.15 and 0.013 , respectively, for the $73^{\circ}$ cone. The indicated dots at the extreme points of the serrated paths are used as input for the Marciniak-Kuczynski code, which further assumes linear paths between these corner points.

Figure 2 shows predicted traditional forming limit curves for the three material models, i.e. assuming monotonic loading paths. A value of 0.998 has been used for the initial ratio between the thickness of the groove and the sheet, which in view of the hardening coefficient $n=0.224$, produces realistic forming limits of about $22 \%$ in monotonic plane strain. The right-hand sides of the forming limit curves are strongly influenced by the adopted yield locus. Lower limit strains are obtained there with the texture-based yield surface, which results from the curvatures of the yield loci in the region between the points of equibiaxial strain and plane strain.

The predicted forming limits for the strains encountered in incremental forming are given in Fig. 3. The lowest forming limits are obtained when monotonic loading in the 
direction of the final total strains is assumed throughout the process (i.e. the linear strain paths indicated by the dashed lines).

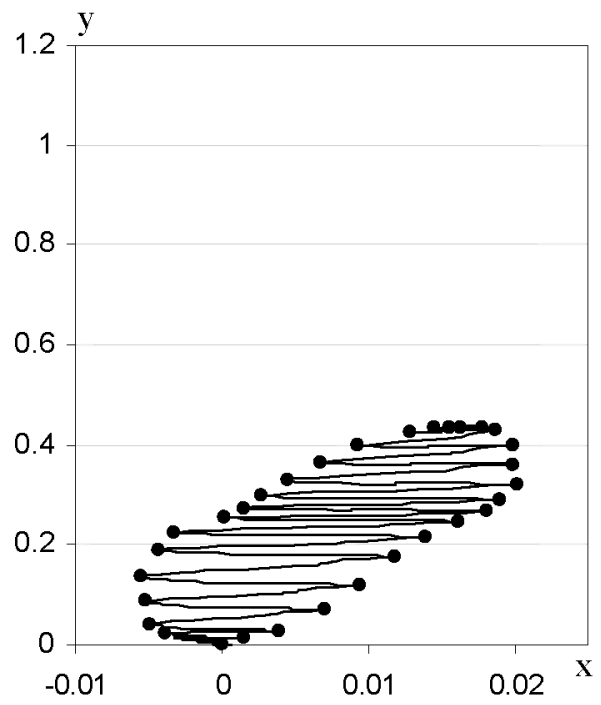

(a)

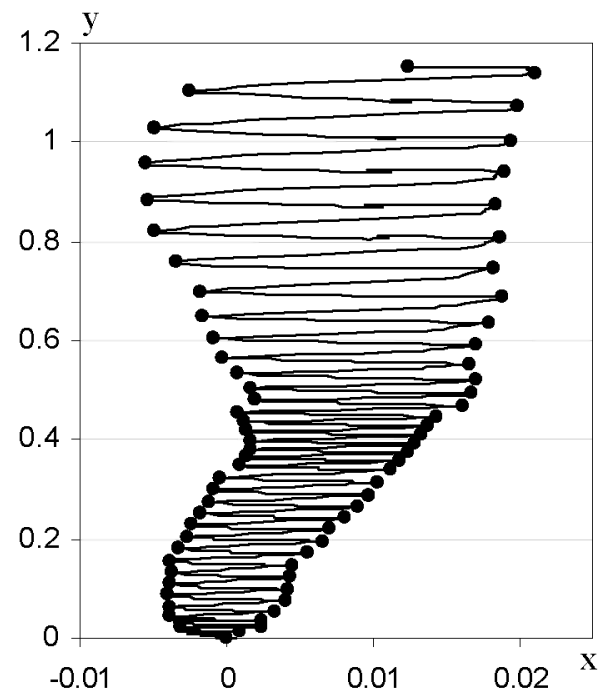

(b)

FIGURE 1. Simulated strain path for cones with wall angles of (a) $50^{\circ}$ and (b) $73^{\circ}$, showing the major and minor true strains in the sheet plane along the $y$ - and the $x$-axis, respectively, in an element of the outer layer across the sheet thickness.

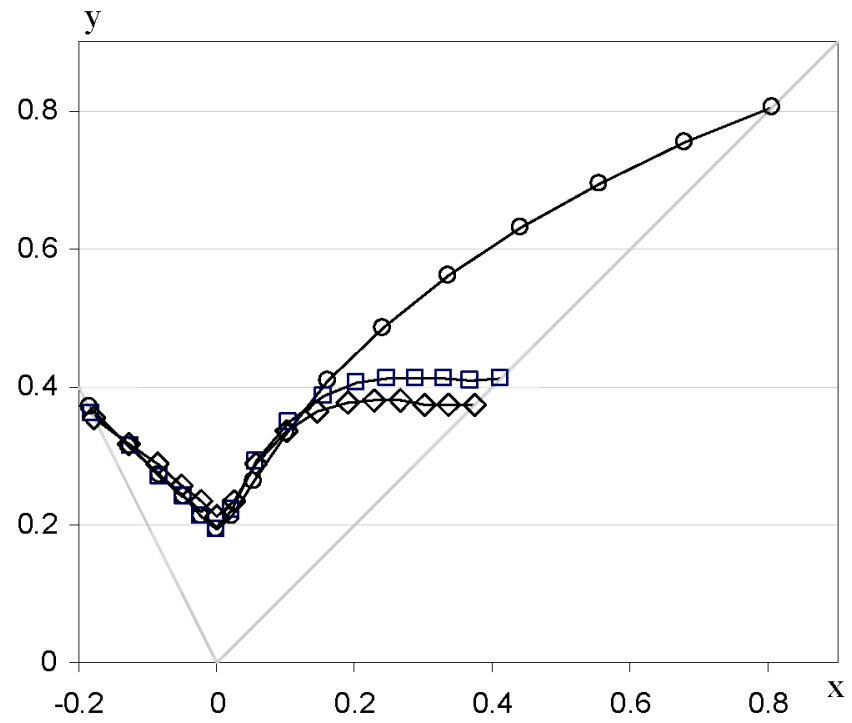

FIGURE 2. Predicted forming limit curves in case of monotonic loading paths for the three material models (circles for VON-ISO, squares for TEX-ISO, diamonds for TEX-MIC). 


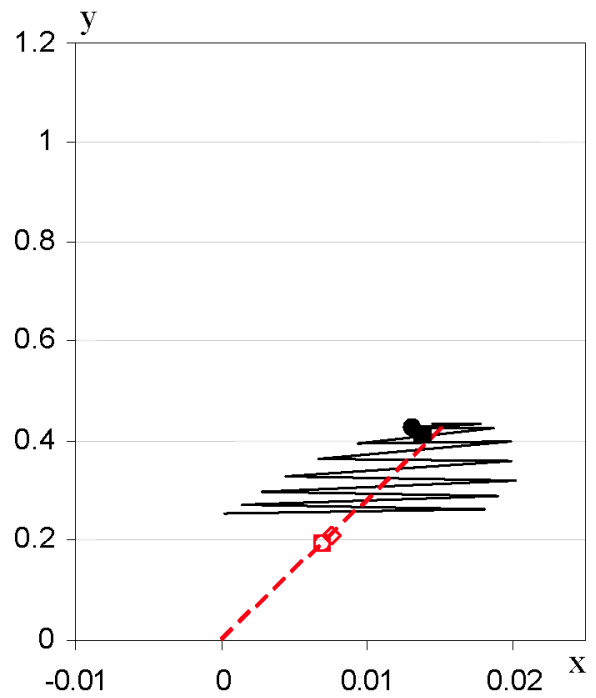

(a)

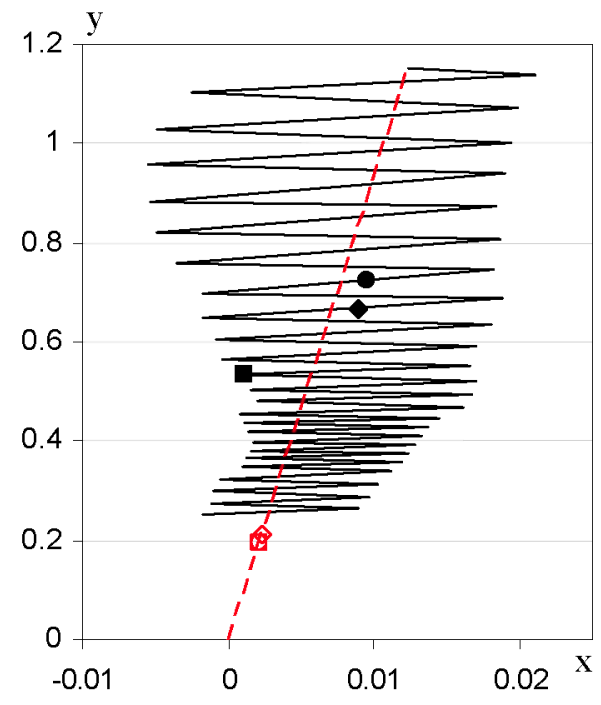

(b)

FIGURE 3. Predicted forming limits for cones with wall angles of (a) $50^{\circ}$ and (b) $73^{\circ}$ for the three material models (circles for VON-ISO, squares for TEX-ISO, diamonds for TEX-MIC). The closed symbols are obtained for the serrated strain paths derived from Fig. 1, the open symbols for the monotonic strain paths indicated by the dashed lines.

For the three material models, the predicted forming limits are considerably higher if the serrated strain paths derived from Fig. 1 are considered instead of monotonic loading. In case of the $50^{\circ}$ cone, necking is predicted at $41 \%$ major true plastic strain for the TEX-Iso model and $43 \%$ for the VON-Iso model, whereas no necking is found in case of the TEX-MIC model. For the $73^{\circ}$ cone, necking is predicted for all three material models, with forming limits of $53 \%, 67 \%$ and $72 \%$ in case of TEX-IsO, TEXMiC and VON-IsO, respectively.

It is clear that a reliable model should predict the absence of necking for the cone with wall angle of $50^{\circ}$, since this is well below the experimentally obtained limiting wall angle of $72^{\circ}$. Such necking is only just avoided with the TEX-Mic model. For the $73^{\circ}$ wall angle, which is only slightly higher than the limiting wall angle, all models predict necking long before the end of the process. This indicates that even taking strain path changes, the texture yield locus and anisotropic hardening into account is insufficient to predict the forming limits in incremental forming.

One of the assumptions in the current Marciniak-Kuczynski model is that the whole sheet is subjected to a uniform deformation. In particular, the strain path for an element in the outer of 3 layers across the sheet thickness is considered. This is the strain history at a depth of about $1 / 6$ of the sheet thickness from the outer surface. In reality, the deformations are not uniform at all across the sheet thickness. For example, the simulations reveal that as the outer layer is elongated along the circumferential direction, the inner layer is compressed, and vice versa. Thereby, a sort of bending and reverse bending occurs twice each time the forming tool passes nearby. It is surmised 
that the corresponding strain gradients across the sheet thickness play an important role for the occurrence of necking.

\section{CONCLUSIONS}

Partial cones with wall angles of $50^{\circ}$ and $73^{\circ}$ have been incrementally formed using aluminium 3003-O of $1.2 \mathrm{~mm}$ thickness, and these processes have been simulated with an implicit elastic-plastic finite-element program. The simulated strain paths at a depth of about $1 / 6$ of the outer sheet surface have been used as input for a MarciniakKuczynski type prediction of forming limits, accounting also for the initial texturebased anisotropy and anisotropic hardening. The predicted forming limits are considerably higher than for monotonic loading, but nevertheless underestimate the experimentally observed formability during incremental forming.

It is expected that this discrepancy is caused by bending and reverse bending effects and corresponding strain gradients across the sheet thickness, which are not taken into account in the present model. It is planned to apply the current approach also for strain paths in the other layers across the sheet thickness, in order to study the necking tendencies at different depths. In reality, the different layers must interact with each other, and it is expected that more accurate necking predictions will require new models that take these interactions into account.

\section{ACKNOWLEDGMENTS}

The authors gratefully acknowledge the financial support by the Federal Government of Belgium (Belgian Science Policy Contract P5/08) and by the Institute for the Promotion of Innovation by Science and Technology in Flanders (IWT). As Research Director of the Fund for Scientific Research (FNRS, Belgium), A.M. Habraken also thanks this research fund for its support.

\section{REFERENCES}

1. J. Jeswiet, F. Micari, G. Hirt, A. Bramley, J. Duflou and J. Allwood, CIRP Annals 54/2, 623-650 (2005).

2. M.S. Shim and J.J. Park, Journal of Materials Processing Technology 113, 654-658 (2001).

3. P. Flores, L. Duchêne, C. Bouffioux, T. Lelotte, C. Henrard, N. Pernin, A. Van Bael, S. He, J. Duflou, A.M. Habraken, International Journal of Plasticity 23, 420-449 (2007).

4. S. He, A. Van Bael and P. Van Houtte, Materials Science Forum 495-497, 1573-1578 (2005).

5. J. Duflou, A. Szekeres and P. Vanherck, Advanced Materials Research 6-8, 441-448 (2005).

6. P. Flores, P. Moureaux, A.M. Habraken, Advances in Experimental Mechanics IV 3-4, 91-97 (2005).

7. P. Van Houtte and A. Van Bael, Int. J. Plasticity 20, 1505-1524 (2004).

8. S. He, A. Van Bael, P. Van Houtte, A. Szekeres, J. Duflou, C. Henrard and A.M. Habraken, Advanced Materials Research 6-8, 525-532 (2005).

9. Z. Marciniak and K. Kuczynski, Int. J. Mech. Sci. 9, p.609 (1967).

10. P. Eyckens, S. He, A. Van Bael, P. Van Houtte and J. Duflou, "Forming limit predictions for the serrated strain paths in single point incremental forming", to be presented at NUMIFORM07. 